\title{
Appel Reaction of Carboxylic Acids with Tribromoisocyanuric Acid/ Triphenylphosphine: a Mild and Acid-Free Preparation of Esters and Amides
}

\author{
Haryadylla da Cunha Sindra and Marcio C. S. de Mattos* \\ Departamento de Química Orgânica, Instituto de Química, Universidade Federal do Rio de Janeiro, \\ CP 68545, 21945-970 Rio de Janeiro-RJ, Brazil
}

\begin{abstract}
A facile and efficient method for esterification and amidation of carboxylic acids under neutral conditions has been developed. Esters and amides can be prepared by reacting a carboxylic acid $(1 \mathrm{mmol})$ with tribromoisocyanuric acid $(0.37 \mathrm{mmol})$ and triphenylphosphine $(1 \mathrm{mmol})$ in dichloromethane at room temperature, followed by addition of an alcohol or an amine, respectively.
\end{abstract}

Keywords: amides, esters, tribromoisocyanuric acid, acylation, triphenylphosphine

\section{Introduction}

Esters and amides are important intermediates in the chemical and pharmaceutical industries. Besides, some of them are bioactive compounds. ${ }^{1}$ In general, their preparations from the corresponding carboxylic acids are well-known transformations and numerous methods have been reported. ${ }^{2}$ However, the coupling reactions of carboxylic acids with nucleophiles require activation of the carboxyl group by its conversion into the most reactive acyl halide. ${ }^{2}$

On the other hand, acid halides are traditionally prepared from carboxylic acids and several common reagents such as thionyl halides, oxalyl halides or phosphorus (oxy) halides. ${ }^{3}$ However, despite their success, there are still some remaining problems using these reagents as they cannot be applied to acid-sensitive substrates due to the high temperature conditions required and the formation of strong harmful corrosive acids by-produced during the process. Furthermore, if the amount of the reagent used is insufficient, acid anhydrides are obtained instead of acyl halides. ${ }^{4}$ Therefore, convenient protocols for the preparation of acid halides under mild conditions is being subject of intensive research. ${ }^{5}$ In this context, the Appel reaction ${ }^{6}$ of carboxylic acids with carbon tetrachloride, ${ }^{7}$ trichloroacetonitrile ${ }^{8}$ or trichloroacetamide ${ }^{9}$ (among others) in the presence of triphenylphosphine $\left(\mathrm{PPh}_{3}\right)$ have been efficiently used for the preparation of acyl chlorides with high efficiency. Despite being the driving force in Appel-type reactions, the formation of the triphenylphosphine oxide by-product can

*e-mail: mmattos@iq.ufrj.br result in an inconvenient purification process; therefore the development of new methods that avoid or minimize the triphenylphosphine oxide by-product in Appel reactions is subject of intensive research. ${ }^{10}$

Trihaloisocyanuric acids [1,3,5-trihalo-1,3,5-triazine2,4,6-(1H,3H,5H)-triones] (Figure 1) are safe, stable and easily handled solids used as electrophilic halogenating reagents. ${ }^{11}$ Although diverse trihaloisocyanuric acids are known, ${ }^{12}$ the most widely used is the inexpensive and commercially available trichloroisocyanuric acid (TCCA; Figure $1, \mathrm{X}=\mathrm{Cl}$ ). While not yet commercially available, tribromoisocyanuric acid (TBCA; Figure 1, $\mathrm{X}=\mathrm{Br}$ ) can be easily prepared from inexpensive materials (cyanuric acid, $\mathrm{KBr}$ and oxone)..$^{13}$<smiles></smiles>

Figure 1. Trihaloisocyanuric acids.

In a pioneering paper, Hiegel et al. ${ }^{14}$ reported the conversion of phenylacetic acid into the acyl chloride using trichloroisocyanuric acid along with triphenylphosphine and subsequent addition of dry methanol produced methyl phenylacetate in $95 \%$ yield. Later, the trichloroisocyanuric acid/triphenylphosphine system was used to convert several carboxylic acids into amides, esters and acyl azides, by reactions with amines, alcohols ${ }^{15}$ and sodium azide, ${ }^{16}$ respectively. 
<smiles>O=c1n(Br)c(=O)n(Br)c(=O)n1Br</smiles>

(1)

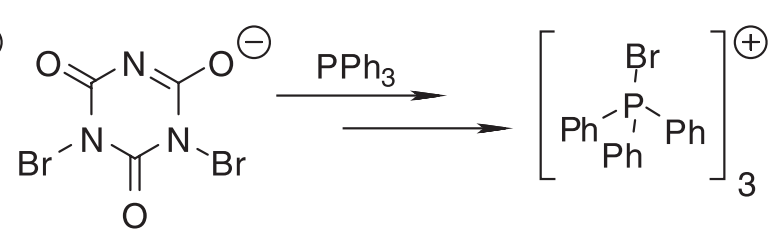

Scheme 1.

Recently, we showed an efficient Appel conversion of alcohols into the corresponding alkyl bromides using tribromoisocyanuric acid/triphenylphosphine system, ${ }^{17}$ being a bromophosphonium salt (1), formed by reaction of triphenylphosphine with TBCA, the key reactive species for such transformation (Scheme 1). ${ }^{17,18}$

In continuation of our studies on the application of tribromoisocyanuric acid in organic synthesis, ${ }^{19}$ herein we report the preparation of esters and amides by reaction of carboxylic acids with alcohols and amines, respectively, in the presence of the tribromoisocyanuric acid/triphenylphosphine system.

\section{Results and Discussion}

The reactions were carried out at room temperature by stirring together the carboxylic acid $(1 \mathrm{mmol})$, tribromoisocyanuric acid $(0.37 \mathrm{mmol})$ and triphenylphosphine $(1 \mathrm{mmol})$ in dichloromethane. The progress of the reaction was followed by gas chromatography with mass spectrometer (GC-MS) and after complete substrate conversion to the acyl bromide (confirmed by MS), an alcohol (1 $\mathrm{mmol}$ ) or an amine (excess) was added to the reaction media. The reactions gave, respectively, esters and amides (along with triphenylphosphine oxide and cyanuric acid), that were isolated by column chromatography and characterized by their melting points (amides) and spectroscopic techniques. In general, the reactions proceeded smoothly and gave products in moderate to excellent yields, showing that the method is quite general and suitable for the conversion of carboxylic acids into their corresponding esters or amides. Tables 1 and 2 show the results.

Interestingly, although tribromoisocyanuric acid is known to be a powerful alcohol oxidant ${ }^{20}$ and electrophilic brominating reagent for alkenes ${ }^{21}$ and arenes, ${ }^{22}$ no brominated or oxidation products were detected by the analytical techniques employed in the crude reaction, indicating a fast formation of bromotriphenylphosphonium salt from the reaction of TBCA with triphenylphosphine.

Based on the above results, a plausible scheme for this transformation (Scheme 2) proceeds through the bromophosphonium salt (1), formed by bromination of triphenylphosphine by TBCA, that is further converted into the oxyphosphonium intermediate (2). Bromide anion attacks on the intermediate $\mathbf{2}$ gives the acyl bromide and triphenylphosphine oxide. Simple nucleophilic addition of the alcohol or the amine to the acyl bromide led to the ester or amide along with isocyanuric acid.

A comparison between our methodology and similar ones previously published (trichloroacetamide $/ \mathrm{PPh}_{3},{ }^{,}$ trichloroacetonitrile/ $\mathrm{PPh}_{3}{ }^{23}$ ethyl tribromoacetate $/ \mathrm{PPh}_{3}{ }^{24}$ or hexabromoacetone $\left./ \mathrm{PPh}_{3}\right)^{25}$ for the preparation of $\mathrm{N}$-cyclohexylbenzamide indicated similar yields (Table 3). However, the advantages of TBCA compared to other reagents used in such transformations is its stability, easy manipulation and preparation and high atom economy, ${ }^{26}$ i.e., percentage of mass transferred to triphenylphosphine to generate the halophosphonium salt (1), the reactive species. Besides, the cyanuric acid by-product formed can be reused to produce more TBCA through a green process (Scheme 3). ${ }^{21}$

\section{Conclusions}

In summary, we have developed a new and convenient route for the preparation of esters and amides from carboxylic acids under neutral conditions. The reaction is easily reproducible, the conditions are mild and the reagents are easily available.

\section{Experimental}

\section{General information}

All chemicals and solvents were used as received. Tribromoisocyanuric acid was prepared as previously described. ${ }^{13}$ Nuclear magnetic resonance (NMR) spectra were recorded on a Bruker AC-200 or AC-300 spectrometers (Bruker, Billerica, MA, USA) at 200 or $300 \mathrm{MHz}\left({ }^{1} \mathrm{H}\right)$ and 50 or $75\left({ }^{13} \mathrm{C}\right)$ using tetramethylsilane (TMS) as internal standard. Gas chromatography were performed on a HP 5890 Series II gas chromatograph (Hewlett-Packard, Palo Alto, CA, USA) with flame ionization detector (FID) using a $30 \mathrm{~m}$ (length), $0.25 \mathrm{~mm}$ (inner diameter), and $25 \mu \mathrm{m}$ (phase thickness) RTX-5 capillary column (Restek Corporation, Bellefonte, PA, USA) and $\mathrm{H}_{2}$ (flow rate $50 \mathrm{~cm} \mathrm{~s}^{-1}$ ) as carrier 
Table 1. Esterification of carboxylic acids promoted by tribromoisocyanuric acid (TBCA)/triphenylphosphine

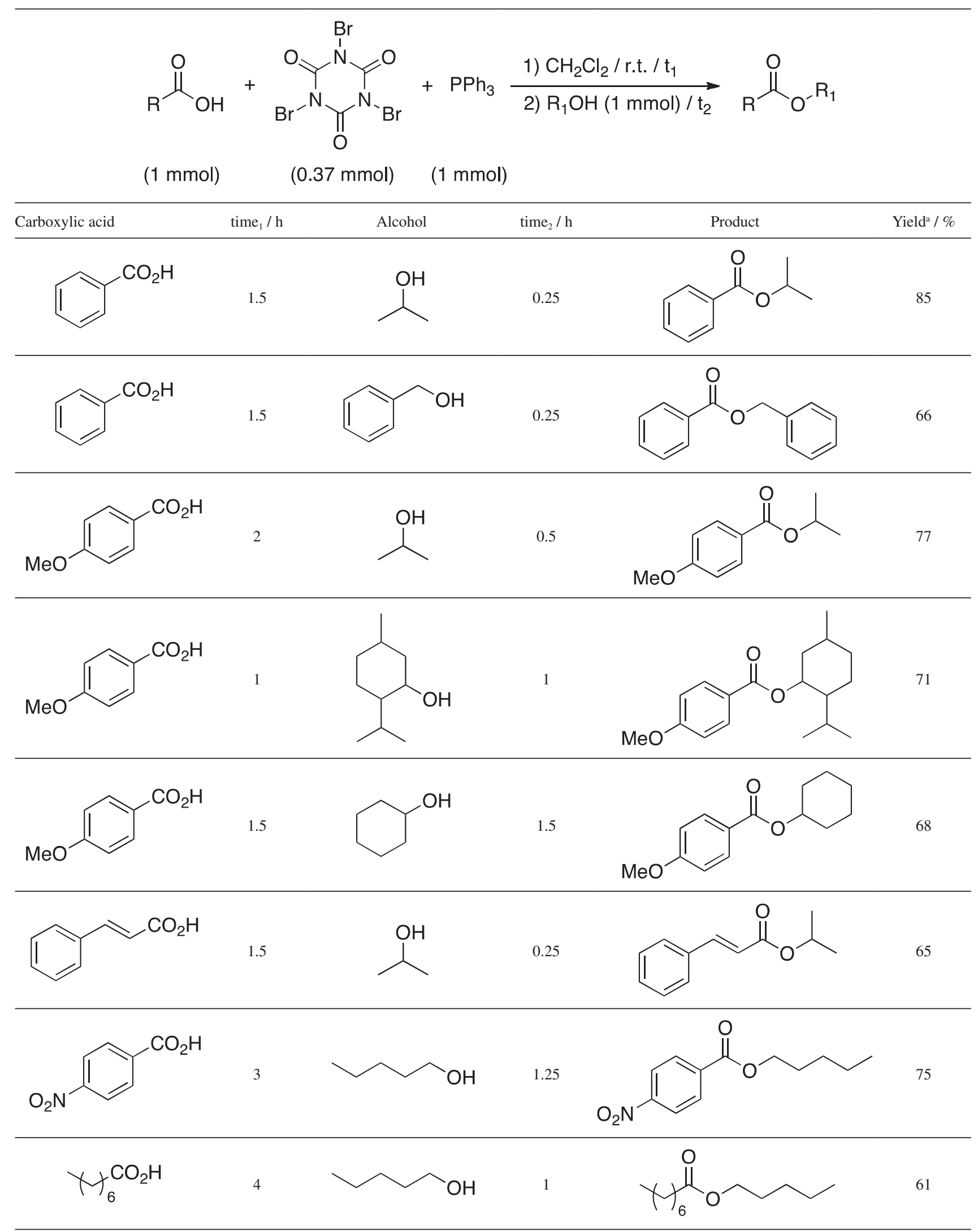


Table 2. Amidation of carboxylic acids promoted by tribromoisocyanuric acid (TBCA)/triphenylphosphine

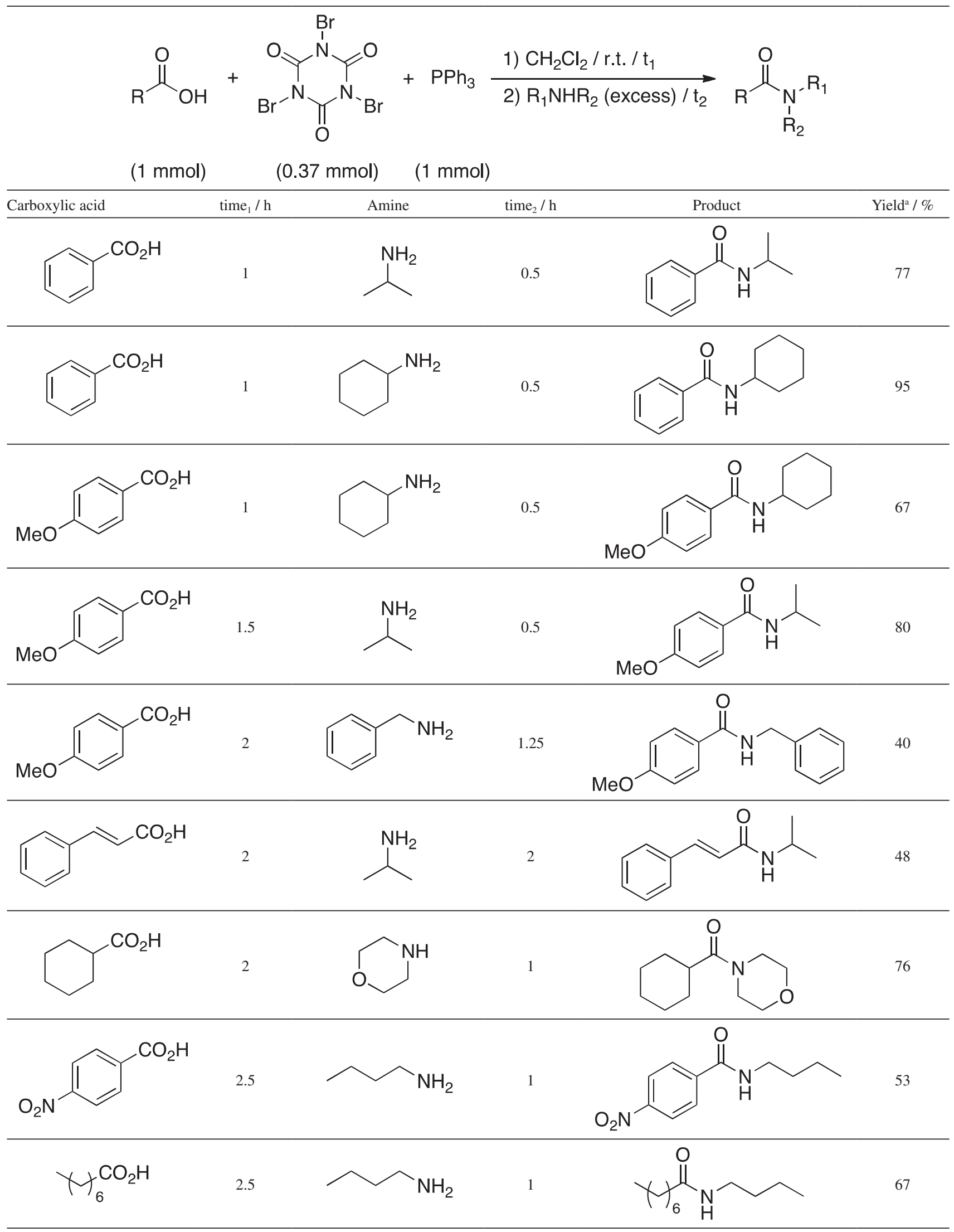

aield of pure product based on carboxylic acid. 


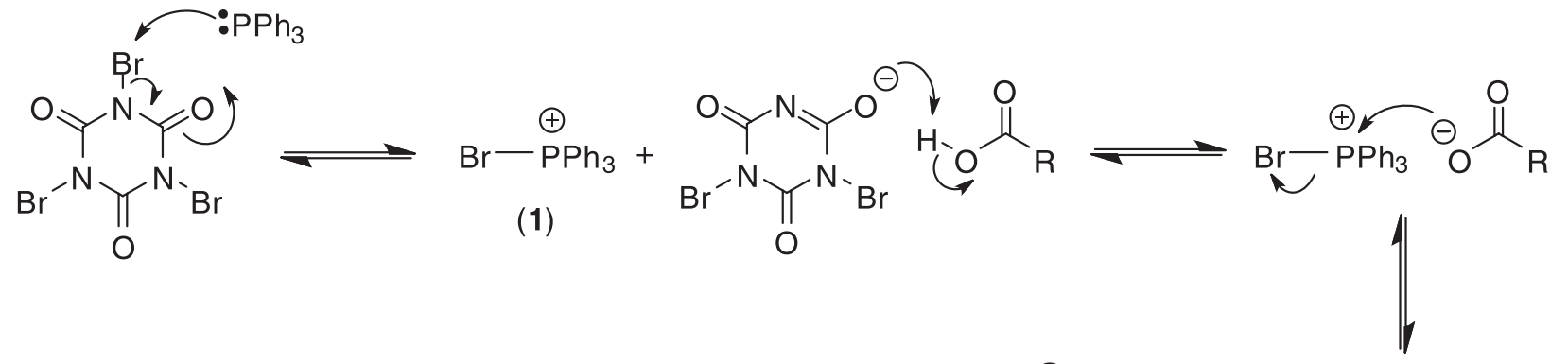

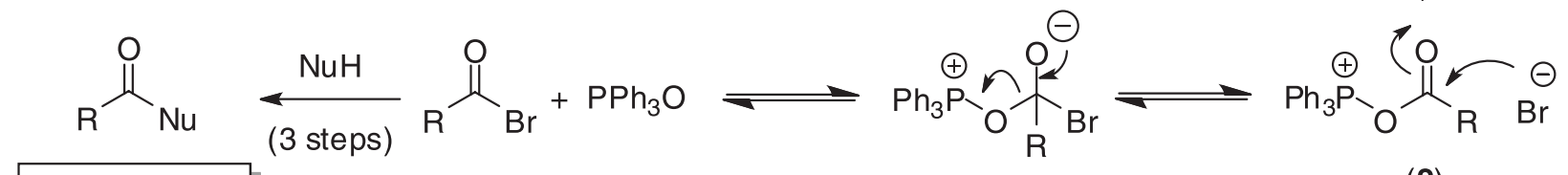
$\mathrm{Nu}=\left\{\begin{array}{l}\mathrm{R}_{1} \mathrm{O} \\ \mathrm{R}_{2} \mathrm{NR}_{3}\end{array}\right.$

Scheme 2.

Table 3. Preparation of $N$-cyclohexylbenzamide<smiles>NC1CCCCC1</smiles>

\begin{tabular}{lccc}
\hline Reagent & Base & Yield / \% & Reference \\
\hline $\mathrm{CCl}_{3} \mathrm{CONH}_{2}$ (2 equiv.), $\mathrm{PPh}_{3}$ (2 equiv.) & 4-picoline (3 equiv.) & 99 & 9 \\
$\mathrm{CCl}_{3} \mathrm{CN}$ (2 equiv.), $\mathrm{PPh}_{3}$ (2 equiv.) & $\mathrm{NEt}_{3}$ (3 equiv.) & 97 & 23 \\
$\mathrm{CBr}_{3} \mathrm{CO}_{2} \mathrm{Et}$ (2 equiv.), $\mathrm{PPh}_{3}$ (2 equiv.) & $\mathrm{NEt}_{3}$ (3 equiv.) & 75 & 24 \\
$\left(\mathrm{CBr}_{3}\right)_{2} \mathrm{CO}$ (0.3 equiv.), $\mathrm{PPh}_{3}$ (1.5 equiv.) & $\mathrm{NEt}_{3}$ (3 equiv.) & 95 & 25 \\
$\mathrm{TBCA}\left(0.37\right.$ equiv.), $\mathrm{PPh}_{3}$ (1 equiv.) & - & this work \\
\hline
\end{tabular}

aAll reactions performed in $\mathrm{CH}_{2} \mathrm{Cl}_{2}$. $\mathrm{PPh}_{3}$ : Triphenylphosphine; TBCA: tribromoisocyanuric acid; $\mathrm{NEt}_{3}$ : triethylamine.

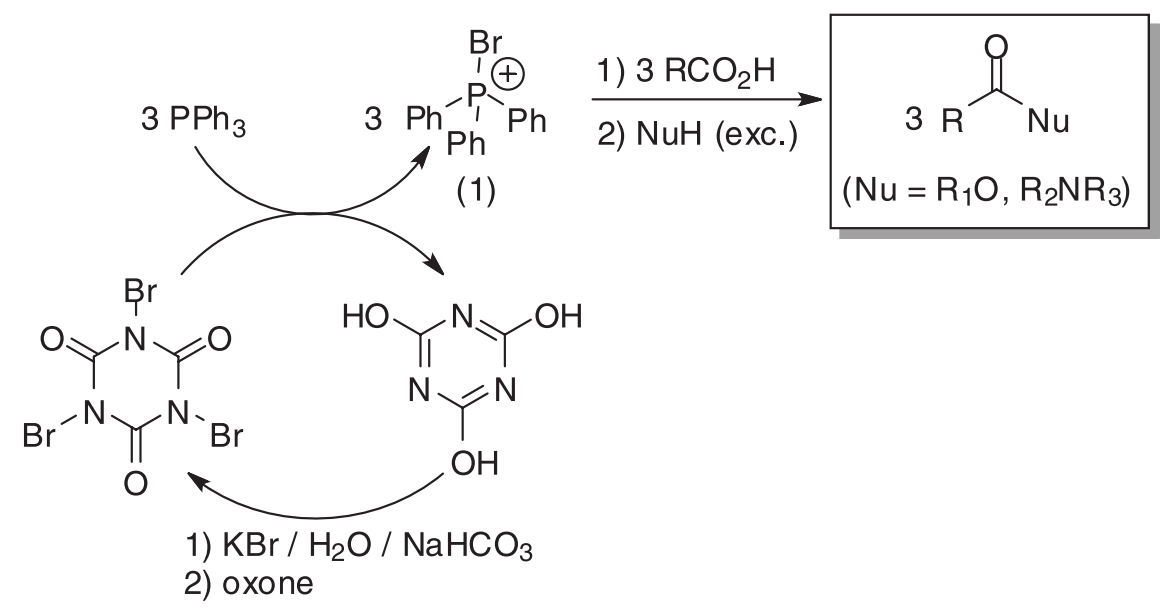

Scheme 3. 
gas (split 1:10). GC-MS analyses were performed on a Shimadzu GCMS-QP2010S gas chromatograph (Shimadzu, Kyoto, Japan) with electron impact $(70 \mathrm{eV})$ by using a $30 \mathrm{~m}$ DB-5 silica capillary column with $0.25 \mathrm{~mm}$ inner diameter and $0.25 \mathrm{~mm}$ phase thickness. Melting points were determined on a Laboratory Device Mel-Temp II (Laboratory Devices, USA) and are corrected.

General procedure for esterification of carboxylic acids promoted by TBCA/triphenylphosphine

To a stirred solution of TBCA $(0.37 \mathrm{mmol})$ and triphenylphosphine $(1 \mathrm{mmol})$ in $\mathrm{CH}_{2} \mathrm{Cl}_{2}\left(6 \mathrm{~cm}^{3}\right)$ was added the carboxylic acid $(1 \mathrm{mmol})$ at room temperature. The progress of the reaction was monitored by GC-MS (time ${ }_{1}$ in Table 1) and, then, alcohol (1 mmol) was added. After time ${ }_{2}$ (Table 1), cyanuric acid was filtered off and the liquid was evaporated on a rotatory evaporator under reduced pressure. The producs were purified by column chromatography (silica gel 70-230 mesh) using diethyl ether/pentane as eluent.

\section{Isopropyl benzoate ${ }^{27}$}

Colorless liquid; ${ }^{1} \mathrm{H}$ NMR (200 MHz, $\left.\mathrm{CDCl}_{3}\right) \delta 1.35$ (d, 6H, $J$ 6.3), 5.16-5.36 (hept, $1 \mathrm{H}, J$ 6.2), 7.38-7.52 (m, $3 \mathrm{H}), 8.05(\mathrm{~m}, 2 \mathrm{H}) ;{ }^{13} \mathrm{C}$ NMR $\left(50 \mathrm{MHz}, \mathrm{CDCl}_{3}\right) \delta 22.0$, 68.4, 128.3, 129.6, 131.0, 132.8, 166.2; MS (70 eV) $\mathrm{m} / \mathrm{z}$ $164\left(\mathrm{M}^{+}\right), 123,105$ (100\%), 77, 59, 51, 43.

\section{Benzyl benzoate ${ }^{28}$}

Colorless liquid; ${ }^{1} \mathrm{H}$ NMR $\left(300 \mathrm{MHz}, \mathrm{CDCl}_{3}\right) \delta 5.40$ (s, 2H), 7.37-7.50 (m, 7H), 7.56-7.61 (m, 1H), 8.10-8.14 $(\mathrm{m}, 2 \mathrm{H}) ;{ }^{13} \mathrm{C}$ NMR $\left(75 \mathrm{MHz}, \mathrm{CDCl}_{3}\right) \delta 66.9,128.3,128.4$, 128.6, 128.8, 129.9, 130.3, 133.2, 136.2, 166.6; MS (70 eV) $\mathrm{m} / \mathrm{z}, 212\left(\mathrm{M}^{+}\right), 194,105(100 \%), 91,77,65,51$.

\section{Isopropyl 4-methoxybenzoate ${ }^{29}$}

Yellowish liquid; ${ }^{1} \mathrm{H}$ RMN ( $\left.300 \mathrm{MHz}, \mathrm{CDCl}_{3}\right) \delta 1.35$ (d, $6 \mathrm{H}, J 6.2$ ), 3.83 (s, 3H), 5.16-5.28 (hept, $1 \mathrm{H}, J 6.2$ ), 6.90 (d, $2 \mathrm{H}, J$ 8.9), 7.98 (d, 2H, J 9.0); ${ }^{13} \mathrm{C} \mathrm{NMR} \mathrm{(75} \mathrm{MHz,} \mathrm{CDCl}_{3}$ ) $\delta$ 22.2, 55.6, 68.1, 113.7, 123.6, 131.7, 163.4, 166.1; MS $(70 \mathrm{eV}) \mathrm{m} / \mathrm{z} 194\left(\mathrm{M}^{+}\right), 179,152,135$ (100\%), 92, 77, 64, 43.

\footnotetext{
Menthyl 4-methoxybenzoate ${ }^{30}$

Yellowish oil; ${ }^{1} \mathrm{H}$ NMR $\left(300 \mathrm{MHz}, \mathrm{CDCl}_{3}\right) \delta 0.80(\mathrm{~d}$, $3 \mathrm{H}, J$ 6.9), 0.91-0.94 (m, 7H), 1,03-1.16 (m, 2H), 1.49-1.59 (m, 2H), 1.70-1.75 (m, 2H), 1.94-2.01 (m, 1H), 2.10-2,14 $(\mathrm{m}, 1 \mathrm{H}), 3.86(\mathrm{~s}, 3 \mathrm{H}), 4.86-4.95(\mathrm{~m}, 1 \mathrm{H}), 6.92(\mathrm{~d}, 2 \mathrm{H}$, $J$ 9.0), 8.01 (d, $2 \mathrm{H}, J$ 8.8); ${ }^{13} \mathrm{C}$ NMR $\left(75 \mathrm{MHz}, \mathrm{CDCl}_{3}\right.$ ) $\delta 16.8,21.0,22.2,23.9,26.7,31.6,34.6,41.3,47.5,55.6$, 74.7, 113.7, 123.5, 131.74, 163.4, 166.1; MS $(70 \mathrm{eV}) \mathrm{m} / \mathrm{z}$ $290\left(\mathrm{M}^{+}\right), 152,135(100 \%), 123,95,81,55,41$.
}

Cyclohexyl 4-methoxybenzoate ${ }^{31}$

Yellowish oil; ${ }^{1} \mathrm{H}$ NMR $\left(300 \mathrm{MHz}, \mathrm{CDCl}_{3}\right) \delta 1.31-1.63$ (m, 6H), 1.76-1.83 (m, 2H), 1.91-1,95 (m, 2H), 3.87 (s, 3H), 4.96-5.04 (m, 1H), 6.91 (d, 2H, J 9.0), 7.99 (d, 2H, J 9.0); ${ }^{13} \mathrm{C}$ NMR $\left(75 \mathrm{MHz}, \mathrm{CDCl}_{3}\right) \delta 23.9,25.7,31.9,55.6,72.9$, 113.7, 123.7, 131.7, 163.4, 166.0; MS (70 eV) m/z 234 $\left(\mathrm{M}^{+}\right), 152,135$ (100\%), 107, 92, 77, 67, 55, 41.

\section{Isopropyl cinnamate ${ }^{32}$}

Yellowish liquid; ${ }^{1} \mathrm{H}$ NMR (300 MHz, $\left.\mathrm{CDCl}_{3}\right) \delta 1.32$ (d, 6H, J 6.7), 5.09-5.22 (hept, 1H, J 6.2), 6.43 (d, 1H, $J 16.0), 7.35-7.39(\mathrm{~m}, 3 \mathrm{H}), 7.49-7.54(\mathrm{~m}, 2 \mathrm{H}), 7.68(\mathrm{~d}, 1 \mathrm{H}$, $J$ 16.0); ${ }^{13} \mathrm{C}$ NMR (75 MHz, $\left.\mathrm{CDCl}_{3}\right) \delta 22.1,67.9,119.0$, 128.1, 129.0, 130.3, 134.7, 144.4, 166.6; MS (70 eV) $\mathrm{m} / \mathrm{z}$ $190\left(\mathrm{M}^{+}\right), 147,131(100 \%), 103,77,51,43$.

\section{Pentyl 4-nitrobenzoate ${ }^{33}$}

Colorless liquid; ${ }^{1} \mathrm{H}$ NMR $\left(300 \mathrm{MHz}, \mathrm{CD}_{3} \mathrm{CN}\right)$ $\delta$ 0.92-0.97 (m, 3H), 1.37-1.48 (m, 4H), 1.74-1.83 (m, 2H), 4.35 (t, 2H, $J$ 6.6), 8.19 (d, 2H, $J$ 9.0), 8.29 (d, 2H, $J 9.0$ ); ${ }^{13} \mathrm{C}$ NMR (75 MHz, $\left.\mathrm{CD}_{3} \mathrm{CN}\right) \delta 12.8,21.6,27.4,27.5,65.3$, 123.1, 130.0, 136.5, 150.1, 164.2; MS (70 eV) $\mathrm{m} / 2.238$ $\left(\mathrm{M}^{+}\right), 168,150,120,104,70$ (100\%), 55, 42.

\section{Pentyl octanoate ${ }^{34}$}

Colorless liquid; ${ }^{1} \mathrm{H}$ NMR $\left(300 \mathrm{MHz}, \mathrm{CD}_{3} \mathrm{CN}\right)$ $\delta$ 0.88-0.95 (m, 6H), 1.31-1.37 (m, 12H), 1.57-1.64 ppm (m, 4H), $2.28(\mathrm{t}, 2 \mathrm{H}, \mathrm{t}, J 7.3), 4.04(\mathrm{t}, 2 \mathrm{H}, J 6.6 \mathrm{~Hz})$; ${ }^{13} \mathrm{C}$ NMR (75 MHz, $\left.\mathrm{CD}_{3} \mathrm{CN}\right) \delta 14.4,14.5,23.2,23.5,26.0$, 29.0, 29.3, 29,9, 29.9, 32.6, 35.0, 65.0, 174.5; MS (70 eV) $\mathrm{m} / \mathrm{z} 214\left(\mathbf{M}^{+}\right), 145,127,70$ (100\%), 57, 55, 43.

General procedure for amidation of carboxylic acids promoted by TBCA/triphenylphosphine

To a stirred solution of TBCA $(0.37 \mathrm{mmol})$ and triphenylphosphine $(1 \mathrm{mmol})$ in $\mathrm{CH}_{2} \mathrm{Cl}_{2}\left(6 \mathrm{~cm}^{3}\right)$ was added the carboxylic acid $(1 \mathrm{mmol})$ at room temperature. The progress of the reaction was monitored by GC-MS (time in Table 2) and, then, the amine $(25-75 \mathrm{mmol})$ was added.

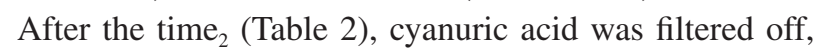
and the liquid was evaporated on a rotatory evaporator under reduced pressure. The producs were purified by column chromatography (silica gel 70-230 mesh) using diethyl ether/pentane as eluent.

\section{$N$-Isopropyl-benzamide}

Yellowish solid; m.p. $94-96{ }^{\circ} \mathrm{C}$ (lit. $95{ }^{\circ} \mathrm{C}$ ) $;{ }^{35}{ }^{1} \mathrm{H}$ NMR (200 MHz, $\mathrm{CDCl}_{3}$ ) $\delta 1.24$ (d, 6H, J 6.5), 4.18-4.35 (sext, $1 \mathrm{H}, J$ 6.6), $6.27(\mathrm{~s}, 1 \mathrm{H}), 7.37-7.45(\mathrm{~m}, 3 \mathrm{H}), 7.74-7.77$ (m, $2 \mathrm{H}) ;{ }^{13} \mathrm{C}$ NMR (50 MHz, $\left.\mathrm{CDCl}_{3}\right) \delta 22.9,42.0,127.0$, 
128.6, 131.3, 135.1, 166.9; MS (70 eV) m/z $163\left(\mathrm{M}^{+}\right), 148$, $105(100 \%), 77,51$.

\section{$\mathrm{N}$-Cyclohexyl-benzamide}

White solid; m.p. $138-140{ }^{\circ} \mathrm{C}$ (lit. $139-141{ }^{\circ} \mathrm{C}$ ); ${ }^{36}$ ${ }^{1} \mathrm{H}$ NMR (300 MHz, $\left.\mathrm{CDCl}_{3}\right) \delta 1.18-2.05(\mathrm{~m}, 10 \mathrm{H}), 3.92-$ $4.04(\mathrm{~m}, 1 \mathrm{H}), 6.06(\mathrm{~s}, 1 \mathrm{H}), 7.38-7.49(\mathrm{~m}, 3 \mathrm{H}), 7.74-7.78$ $(\mathrm{m}, 2 \mathrm{H}) ;{ }^{13} \mathrm{C}$ NMR $\left(75 \mathrm{MHz}, \mathrm{CDCl}_{3}\right) \delta 25.1,25.8,33.4$, 48.9, 127.0, 128.7, 131.4, 135.3, 166.8.; MS $(70 \mathrm{eV}) \mathrm{m} / \mathrm{z}$ $203\left(\mathrm{M}^{+}\right), 123,122,105(100 \%), 77,51$.

\section{$\mathrm{N}$-Cyclohexyl-4-methoxybenzamide}

White solid; m.p. 153-155 ${ }^{\circ} \mathrm{C}$ (lit. 153-154 ${ }^{\circ} \mathrm{C}$ ); ${ }^{37}$ ${ }^{1} \mathrm{H} \mathrm{NMR}\left(300 \mathrm{MHz}, \mathrm{CDCl}_{3}\right) \delta 1.16-1.29$ (m, 3H), 1.35-1.50 $(\mathrm{m}, 2 \mathrm{H}), 1.62-1.79(\mathrm{~m}, 3 \mathrm{H}), 2.02-2.06(\mathrm{~m}, 2 \mathrm{H}), 3.84(\mathrm{~s}, 1 \mathrm{H})$, 3.91-4.03 (m, 1H), $5.93(\mathrm{~s}, 1 \mathrm{H}), 6.91(\mathrm{~d}, 2 \mathrm{H}, J$ 8.9), 7.72 $\left(\mathrm{d}, 2 \mathrm{H}, J\right.$ 8.8); ${ }^{13} \mathrm{C}$ NMR $\left(75 \mathrm{MHz}, \mathrm{CDCl}_{3}\right) \delta 25.1,25.8$, 33.5, 48.8, 55.6, 113.9, 127.6, 128.8, 162.2, 166.3; MS $(70 \mathrm{eV}) \mathrm{m} / \mathrm{z} 233\left(\mathrm{M}^{+}\right), 232(2,49), 152,151,135(100 \%)$, $107,92,79,77$.

\section{$\mathrm{N}$-Isopropyl-4-methoxybenzamide}

White solid; m.p. 112-114 ${ }^{\circ} \mathrm{C}$ (lit. $123^{\circ} \mathrm{C}$ ) $;^{35}{ }^{1} \mathrm{H}$ NMR $\left(300 \mathrm{MHz}, \mathrm{CDCl}_{3}\right) \delta 1.25(\mathrm{~d}, 6 \mathrm{H}, J 6.3), 3.83(\mathrm{~s}, 3 \mathrm{H})$, 4.21-4.33 (m, 1H), $6.00(\mathrm{~s}, 1 \mathrm{H}), 6.89$ (d, 2H, J 8.9), 7.73 $\left(\mathrm{d}, 2 \mathrm{H}, J\right.$ 8.9); ${ }^{13} \mathrm{C}$ NMR $\left(75 \mathrm{MHz}, \mathrm{CDCl}_{3}\right) \delta 23.0,41.9$, $55.5,113.8,127.3,128.8,162.1,166.4$; MS $(70 \mathrm{eV}) \mathrm{m} / \mathrm{z}$ $193\left(\mathrm{M}^{+}\right), 135$ (100\%), 107, 104, 92, 77.

\section{$\mathrm{N}$-Benzyl-4-methoxybenzamide}

White solid; m.p. $120-122{ }^{\circ} \mathrm{C}$ (lit. 124-126 ${ }^{\circ} \mathrm{C}$ ); $;^{38}$ ${ }^{1} \mathrm{H}$ NMR $\left(300 \mathrm{MHz}, \mathrm{CD}_{3} \mathrm{CN}\right) \delta 2.42(\mathrm{~s}, 1 \mathrm{H}), 3.83(\mathrm{~s}$, $3 \mathrm{H}$ ), 4.53 (d, 2H, J 6.1), 6.97 (d, 2H, J 8.9), 7.23-7.35 (m, $5 \mathrm{H}), 7.49(\mathrm{~s}, 1 \mathrm{H}), 7.81\left(\mathrm{~d}, 2 \mathrm{H}, J\right.$ 8.9); ${ }^{13} \mathrm{C}$ NMR $(75 \mathrm{MHz}$, $\left.\mathrm{CD}_{3} \mathrm{CN}\right) \delta 44.3,56.5,115.0,128.3,128.7,129.8,130.3$, 141.2, 163.6, 167.9; MS (70 eV) m/z $241\left(\mathrm{M}^{+}\right), 135(100 \%)$, 107, 92, 77, 64, 51.

\section{$\mathrm{N}$-Isopropyl-cinnamamide}

Yellowish solid; m.p. $94-96{ }^{\circ} \mathrm{C}$ (lit. 101-103 ${ }^{\circ} \mathrm{C}$ ); ${ }^{39}$ ${ }^{1} \mathrm{H}$ NMR (300 MHz, $\left.\mathrm{CDCl}_{3}\right) \delta 1.22(\mathrm{~d}, 6 \mathrm{H}, J$ 6.6), 4.24 (sext, 1H, J 6.5), 5.94 (s, 1H), 6.43 (d, 1H, J 15.6), 7.317.33 (m, 3H), 7.46-7.49 (m, 2H), 7.62 (d, 1H, J 15.3); ${ }^{13} \mathrm{C}$ NMR $\left(75 \mathrm{MHz}, \mathrm{CDCl}_{3}\right) \delta 23.0,41.8,121.4,128.0$, 129.0, 129.7, 135.2, 140.9, 165.4; MS (70 eV) m/z 189 $\left(\mathrm{M}^{+}\right), 174,161,146,131$ (100\%), 118, 112, 103, 91, 87, $77,63,58,51,43$.

\section{$\mathrm{N}$-(Cyclohexylcarbonyl)-morpholine $\mathrm{e}^{40}$}

Yellowish oil; ${ }^{1} \mathrm{H}$ NMR $\left(300 \mathrm{MHz}, \mathrm{CDCl}_{3}\right) \delta 1.21-1.82$ (m, $10 \mathrm{H}), 2.37-2.47$ (m, $1 \mathrm{H}), 3.55-3.68(\mathrm{~m}, 8 \mathrm{H}) ;{ }^{13} \mathrm{C} \mathrm{NMR}$
(75 MHz, $\left.\mathrm{CDCl}_{3}\right) \delta 26.0,29.5,40.4,42.1,46.1,67.1,174.9$; MS (70 eV) m/z $197\left(\mathbf{M}^{+}\right), 142,129,83$ (100\%), 70, 55, 41.

\section{N-Butyl-4-nitrobenzamide}

Yellowish solid; m.p. $102-104{ }^{\circ} \mathrm{C}$ (lit. 104-104.5 ${ }^{\circ} \mathrm{C}$ ) ${ }^{41}$ ${ }^{1} \mathrm{H}$ NMR (300 MHz, $\left.\mathrm{CDCl}_{3}\right) \delta$ 0.92-0.97 (t, 3H, $J$ 7.3), 1.40 (sext, $2 \mathrm{H}, J 7.2$ ), 1.61 (quint, $2 \mathrm{H}, J 7.5$ ), 3.45 (q, 2H, $J 7.2), 6.60$ (s, $1 \mathrm{H}), 7.92$ (d, 2H, J 9.0), 8.24 (d, 2H, J 9.0); ${ }^{13} \mathrm{C} \mathrm{NMR}\left(75 \mathrm{MHz}, \mathrm{CDCl}_{3}\right) \delta 13.2,19.6,31.1,39.7,123.2$, 127.6, 140.0, 149.0, 165.1; MS (70 eV) $\mathrm{m} / \mathrm{z} 222\left(\mathrm{M}^{+}\right), 193$, 180, $150(100 \%), 120,104,92,76,50,41$.

\section{$N$-Butyl-octanamide ${ }^{42}$}

${ }^{1} \mathrm{H}$ NMR $\left(300 \mathrm{MHz}, \mathrm{CDCl}_{3}\right) \delta$ 0.83-0.92 (m, 6H), 1.26-1.36 (m, $10 \mathrm{H}), 1.41-1.49(\mathrm{~m}, 2 \mathrm{H}), 1.57-1.62$ (m, $2 \mathrm{H}), 2.14$ (t, 2H, J 7.4), 3.22 (q, 2H, J 7.2), 5.72 (s, 1H); ${ }^{13} \mathrm{C} \mathrm{NMR}\left(75 \mathrm{MHz}, \mathrm{CDCl}_{3}\right) \delta 13.2,13.5,19.6,22.1,25.4$, 28.5, 28.8, 31.2, 36.4, 38.7, 172.9; MS (70 eV) $\mathrm{m} / z .199$ $\left(\mathrm{M}^{+}\right), 170,156,142,128,115$ (100\%), 100, 86, 73, 57, $44,41$.

\section{Supplementary Information}

${ }^{1} \mathrm{H}$ NMR, ${ }^{13} \mathrm{C}$ NMR and MS spectra of synthesized compounds are available free of charge at http://jbcs.sbq.org.br.

\section{Acknowledgments}

We thank CNPq and FAPERJ for the finacial support.

\section{References}

1. Otera, J.; Nishikido, J.; Esterification: Methods, Reactions, and Applications; Wiley-VCH Verlag GmbH \& Co. KGaA: Weinheim, 2003; Negwer, M.; Scharnow, H.-G.; OrganicChemical Drugs and Their Synonyms, $8^{\text {th }}$ ed.; Wiley-VCH Verlag GmbH \& Co. KGaA: Weinheim, 2001; Pattabiraman, V. R.; Bode, J. W.; Nature (London, U. K.) 2011, 480, 471.

2. Larock, R. C.; Comprehensive Organic Transformations, a Guide to Functional Group Preparation, $2^{\text {nd }}$ ed.; John Wiley \& Sons: New York, 1999; Trost, B. M.; Comprehensive Organic Synthesis: Selectivity, Strategy and Efficiency in Modern Organic Synthesis, vol. 6; Pergamon Press: Oxford, 1991.

3. Ansell, M. F. In The Chemistry of Acyl Halides; Patai, S.; ed.; Interscience: London, 1972, ch. 2; McMaster, L.; Ahmann, F. F.; J. Am. Chem. Soc. 1928, 50, 145; Chen, H.; Xu, X.; Liu, L.; Tang, G.; Zhao, Y.; RSC Adv. 2013, 3, 16247.

4. Adams, R.; Ulich, L. H.; J. Am. Chem. Soc. 1920, 42, 599.

5. Bahrami, K.; Khodaei, M. M.; Targhan, H.; Arabi, M. S.; Tetrahedron Lett. 2013, 54, 5064. 
6. de Andrade, V. S. C.; de Mattos, M. C. S.; Curr. Org. Synth. 2015, 12, 309.

7. Lee, J. B.; J. Am. Chem. Soc. 1966, 88, 3340; Tömösközi, I.; Gruber, L.; Radics, L.; Tetrahedron Lett. 1975, 16, 2473.

8. Jang, D. O.; Park, D. J.; Kim, J.; Tetrahedron Lett. 1999, 40, 5323.

9. Chaysripongkul, S.; Pluempanupat, W.; Jang, D. O.; Chavasiri, W.; Bull. Korean Chem. Soc. 2009, 30, 2066.

10. For selected examples, see: Tang, X.; An, J.; Denton, R. M.; Tetrahedron Lett. 2014, 55, 799; Kosal, A. D.; van Kalkeren, H. A.; Blom, A. L.; Rutjes, F. P. J. T.; Huijbregts, M. A.; Green Chem. 2013, 15, 1255; Byren, P. A.; Rajendran, K. V.; Muldoon, J.; Guilheany, D. G.; Org. Biomol. Chem. 2012, 10, 3531; Wilson, E. E.; Ashfeld, B. L.; Angew. Chem., Int. Ed. 2012, 51, 12036; Rao, A. N.; Ganesan, K.; Shinde, C. K.; Synth. Commun. 2012, 42, 2299; O’Brien, C. J.; Tellez, J. L.; Nixon, Z. S.; Kang, L. J.; Carter, A. L.; Kunkele, S. R.; Przeworski, K. C.; Chass, G. A.; Angew. Chem., Int. Ed. 2009, 48, 6863.

11. Mendonça, G. F.; de Mattos, M. C. S.; Curr. Org. Synth. 2013, 10,820 .

12. Gottardi, W.; Monatsh. Chem. 1967, 98, 507; Gottardi, W.; Monatsh. Chem. 1970, 101, 655; Ribeiro, R. S.; Esteves, P. M.; de Mattos, M. C. S.; Tetrahedron Lett. 2007, 48, 8747; de Almeida, L. S.; Esteves, P. M.; de Mattos, M. C. S.; Synlett 2007, 11, 1687; Ribeiro, R. S.; Esteves, P. M.; de Mattos, M. C. S.; J. Braz. Chem. Soc. 2012, 23, 228.

13. de Almeida, L. S.; Esteves, P. M.; de Mattos, M. C. S.; Synlett 2006, 10, 1515.

14. Hiegel, G. A.; Ramírez, J.; Barr, R. K.; Synth. Commun. 1999, 29, 1415.

15. Rodrigues, R. C.; Barros, I. M. A.; Lima, E. L. S.; Tetrahedron Lett. 2005, 46, 5945.

16. Akhlaginia, B.; Rouhi-Saadabad, H.; Can. J. Chem. 2013, 91, 181.

17. de Andrade, V. S. C.; de Mattos, M. C. S.; J. Braz. Chem. Soc. 2014, 25, 975.

18. Appel, R.; Angew. Chem., Int. Ed. 1975, 14, 801.

19. de Almeida, L. S.; Esteves, P. M.; de Mattos, M. C. S.; Curr. Green Chem. 2014, 1, 94.

20. Crespo, L. T. C.; de Mattos, M. C. S.; Esteves, P. M.; Quim. Nova 2013, 36, 320.

21. Tozetti, S. D. F.; de Almeida, L. S.; Esteves, P. M.; de Mattos, M. C. S.; J. Braz. Chem. Soc. 2007, 18, 675; Crespo, L. T. C.; Ribeiro, R. S.; de Mattos, M. C. S.; Esteves, P. M.; Synthesis 2010, 14, 2379.
22. de Almeida, L. S.; Esteves, P. M.; de Mattos, M. C. S.; Synthesis 2006, 13, 221; de Almeida, L. S.; Esteves, P. M.; de Mattos, M. C. S.; Tetrahedron Lett. 2009, 50, 3001; de Almeida, L. S.; de Mattos, M. C. S.; Esteves, P. M.; Synlett 2013, 24, 603.

23. Jang, D. O.; Park, D. J.; Kim, J.; Tetrahedron Lett. 1999, 40, 5323.

24. Kang, D. H.; Joo, T. Y.; Lee, E. H.; Chaysripongkul, S.; Chavasiri, W.; Jang, D. O.; Tetrahedron Lett. 2006, 47, 5693.

25. Menezes, F. G.; Kolling, R.; Bertoluzzi, A. J.; Gallardo. H.; Zucco, C.; Tetrahedron Lett. 2009, 50, 2559.

26. Trost, B. M.; Science (Washington, DC, U. S.) 1991, 254, 1471.

27. Kim, B. R.; Sung, G. H.; Lee, S.-G.; Yoon, Y. J.; Tetrahedron 2013, 69, 3234.

28. Li, L.; Sheng, H.; Xu, F.; Shen, Q.; Chin. J. Chem. 2009, 27, 1127.

29. Wu, X.-F.; Darcel, C.; Eur. J. Org. Chem. 2009, 8, 1144.

30. Akiyama, F.; Tokura, N.; Bull. Chem. Soc. Jpn. 1966, 39, 131.

31. Kleinpeter, E.; Bölke, U.; Frank, A.; Tetrahedron 2008, 64, 10014.

32. Jia, X.-S.; Wang, H.-L.; Huang, Q.; Kong, L.-L.; Zhang, W.-H.; J. Chem. Res. 2006, 2, 135.

33. Xie, F.; Yan, F.; Chen, M.; Zhang, M.; RSCAdv. 2014, 4, 29502.

34. Giang, L.; Xinzong, L.; Eli, W.; New J. Chem. 2007, 31, 348.

35. Rahman, O.; Kihlberg, T.; Lngstroem, B.; J. Org. Chem. 2003, $68,3558$.

36. Jo, Y.; Ju, J.; Choe, J.; Song, K. H.; Lee, S.; J. Org. Chem. 2009 , $74,6358$.

37. Pelletier, G.; Bechara, W. S.; Charette, A. B.; J. Am. Chem. Soc. 2010, 132, 12817.

38. Fairfull-Smith, K. E.; Jenkins, I. D.; Loughlin, W. A.; Org. Biomol. Chem. 2004, 2, 1979.

39. Huang, Z.; Wen, L.; Huang, X.; Synth. Commun. 1990, 20, 2579.

40. Glynn, D.; Bernier, D.; Woodward, S.; Tetrahedron Lett. 2008, 49, 5687.

41. Connors, K. A.; Bender, M. L.; J. Org. Chem. 1961, 26, 2498.

42. Kunishima, M.; Kikuchi, K.; Kawai, Y.; Hioki, K.; Angew. Chem., Int. Ed. 2012, 51, 2080.

Submitted: October 21, 2015 Published online: January 15, 2016 